\title{
Anomalías congénitas del riñón y del tracto urinario en niños con síndrome de Down
}

\author{
Congenital anomalies of the kidney and the urinary tract in Down syndrome children
}

\author{
Víctor Manuel Mora-Bautista ${ }^{1}$ \\ ${ }^{1}$ Médico y cirujano, Universidad Industrial de Santander. Especialista en pediatría \\ de la Universidad Industrial de Santander. Miembro del Grupo de Síndrome de Down de Santander, Colombia
}

\begin{abstract}
Resumen
Las anomalías del riñón y el tracto urinario hacen parte de las anomalías congénitas relacionadas con el síndrome de Down. No existen recomendaciones específicas. Para el presente estudio, se hizo una revisión por palabras clave en bases de datos (Pubmed, Science Direct, Ovid, Google académico, UpToDate). Se encontró que, en niños con síndrome de Down, las enfermedades congénitas del riñón y el tracto urinario abarcan glomerulonefritis, agenesia renal, microquistes, riñones ectópicos, hidronefrosis, hidrouréter, valvas uretrales posteriores, obstrucción de la uretra anterior e hipospadias. Con respecto a los procesos diagnósticos, sería razonable realizar una ecografía renal durante la primera semana de vida. Adicionalmente, la uretrocistografía sería útil solo en casos seleccionados. Si hay un historial de incontinencia urinaria o de infecciones urinarias de repetición; o se detecta un reflujo vesicoureteral, o caída de la tasa de filtración glomerular estimada, debería considerarse la existencia de una disfunción vesical asociada y podría ser pertinente una evaluación urológica (uroflujometría o urodinamia). Sería recomendable hacer un seguimiento clínico anual de la función renal.
\end{abstract}

Palabras clave: síndrome de Down, enfermedades congénitas, enfermedades renales, enfermedades urológicas, diagnóstico por imagen.

doi: http://dx.doi.org/10.22265/acnef.5.2.268

\begin{abstract}
Congenital anomalies of the kidney and the urinary tract are congenital diseases related to Down syndrome. There are not specific recommendations. A literature review was made using key words through scientific databases (Pubmed, Science Direct, Ovid, Scholar Google, UpToDate). CAKUT in Down syndrome include glomerulonephritis, kidney agenesis, microcysts, ectopic kidneys, hydronephrosis and hydroureter, even posterior urethral valves and anterior urethra obstruction, and hypospadias. It will be feasible thinking about performing kidney and urinary tract ultrasonography in first week of life. Urethrocystography must be done in selected cases. If urinary incontinence exists, patient has a history of urinary tract infections, vesicoureteral reflux has been diagnosed or if a decrease in glomerular filtration rate has been identified, we should check for vesical dysfunction associated to symptoms and urologic evaluation could be needed (uroflowmetry or urodynamics). It might be adequate an annual clinical follow up of kidney function.
\end{abstract}

Key words: Down syndrome, CAKUT, diagnostic imaging.

doi: http://dx.doi.org/10.22265/acnef.5.2.268

Referenciar este artículo: Mora Bautista VM. Rev. Colomb. Nefrol. 2018;5(1): 54-60 doi: http://dx.doi.org/10.22265/acnef.5.2.268

*Correspondencia: vmoramd@medicos.com

Recibido: $17-04-17 \cdot$ Aceptado: 21-08-17 • Publicado en línea: 23-11-17 


\section{Introducción}

$\mathrm{E}$ 1 síndrome de Down es la cromosomopatía más frecuente, que involucra varios mecanismos en los que existen tres copias (trisomía), completas o parciales (región crítica), del cromosoma 21. Su incidencia es de 1,0-1,1 por cada 1.000 nacidos vivos, según la OMS ${ }^{1}$. En Latinoamérica, ésta es de 1,88; y en Sudamérica, de 2,9 por cada 1.000 nacidos vivos ${ }^{2}$. En Colombia, los datos disponibles apunten a una incidencia de 0,51,5 por cada 1.000 nacidos vivos ${ }^{3,4}$.

Las anomalías congénitas del riñón y del tracto urinario (CAKUT en inglés) son un grupo variado de malformaciones ocasionadas por alteraciones en la embriología renal. Su etiología es multifactorial y representan entre el $15 \%$ y el $20 \%$ de las anomalías documentadas prenatalmente, con una tasa de 0,31,3 por cada 1.000 recién nacidos. Son la primera causa de falla renal en la niñez $z^{5}$ y tienen una prevalencia estimada en Colombia de $0,2-0,4 \%{ }^{6}$.

Las patologías del grupo CAKUT hacen parte de las malformaciones relacionadas con el síndrome de Down. Tienen una prevalencia estimada de 2,3-3,2 $\%$, la cual es cerca de cinco veces mayor a la que se conoce en niños sin la cromosomopatía ${ }^{7,8,9}$. Aún más, a largo plazo, el compromiso del tracto urinario puede elevarse hasta un $27 \%$, entre enfermedades congénitas y adquiridas ${ }^{10}$.

Estas enfermedades son importantes, a pesar de que no sean las que causen mayor morbimortalidad a corto plazo (este lugar lo ostentan las patologías cardiorrespiratorias y gastrointestinale ${ }^{11}$ ). A pesar de lo anterior, no existen recomendaciones actuales que las hagan parte del grupo de patologías de vigilancia obligatoria en las guías mundiales de síndrome de Down ${ }^{12,13}$.

Por estos motivos, en el presente documento se pretendió recopilar la información más reciente acerca del beneficio potencial de un manejo anticipatorio específico.

\section{Metodología}

Se hizo una revisión por palabras clave en las siguientes bases de datos: Pubmed, Science Direct, Ovid, Google académico y UpToDate. Se usaron las siguientes palabras clave: síndrome de Down y enfermedad renal, sindrome de Down y enfermedad urológica, síndrome de Down y glomerulonefritis, síndrome de Down y falla renal. Se recurrió también a referencias cruzadas. Se restringió la ventana de búsqueda a los artículos disponibles en los últimos 20 años, que correspondieran a capítulos de libros, revisiones de tema, reportes de casos, series de casos y cohortes.

\section{Epidemiología}

En el síndrome de Down, el grupo CAKUT abarca glomerulonefritis, agenesia renal, microquistes, riñones ectópicos, anormalidades de las vías urinarias con hidronefrosis e hidrouréter, valvas uretrales posteriores y obstrucción de la uretra anterior, además de hipospadias ${ }^{7,13,14}$. Sin discriminar por patología subyacente, se conoce que hasta un 4,5\% de los pacientes con síndrome de Down puede padecer una enfermedad renal crónica ${ }^{15}$.

La enfermedad glomerular suele presentarse entre la segunda y tercera década de la vida. Las patologías más frecuentes son la nefropatía por IgA y la glomeruloesclerosis focal y segmentaria ${ }^{16,17,18,19}$. Se ha planteado que la disfunción inmune asociada al síndrome de Down puede predisponer a los niños a la glomerulonefritis postinfecciosa, pero los datos disponibles son pocos ${ }^{16,20,21}$. Podría haber una frecuencia más alta de glomerulonefritis asociada a anticuerpos ANCA que en niños sin el síndrome ${ }^{19,22}$.

En relación con los quistes, se ha descrito un aumento significativo de la incidencia tanto de micro como de macroquistes, pero la importancia clínica de esta diferencia es desconocida ${ }^{13}$. La enfermedad renal poliquística autosómica recesiva podría ser más frecuente en niños con síndrome de Down, debido a su gen ubicado en el cromosoma $21^{23}$. 
Genera inquietud que hasta un $4 \%$ de los casos de valvas uretrales posteriores puede corresponder a niños con síndrome de Down. Esto hace pensar que se subestima la uropatía obstructiva en esta población específica, aunque no se observa claramente esta diferencia con las uropatías obstructivas en general $1^{13,24,25,26,27}$. Se ha reportado alteración del desarrollo prostático con consecuencias similares a las valvas uretrales ${ }^{9}$. Además, se tiene conocimiento de la presencia fenotipos de abdomen en ciruela pasa (prune belly) asociados a la trisomía 21 , incluso en niñas ${ }^{28}$.

La hipoplasia o agenesia renal unilateral parece también ser más frecuente en los niños con síndrome de Down; caso que no se ve en las hipospadias o epispadias $^{13}$.

A partir de lo anterior, se considera que la mayoría de hallazgos son alteraciones menores, pero se perciben bastante heterogéneos ${ }^{15}$.

Es de esperar que la proporción de enfermedad renal crónica aumente a largo plazo, dado que hay una mayor supervivencia de estas personas ${ }^{29}$.

En relación con el compromiso funcional, se sabe que podría haber afectación urodinámica hasta en $30 \%$ de los niños ${ }^{30}$ y en $8,7 \%$ de los adultos con la condición ${ }^{31}$. Aunque se sabe que los niños con síndrome de Down tardan más en desarrollar el control de esfínteres (4-5 años), que las niñas tienden a ser más continentes y que puede haber incontinencia en mayor medida (12-16\%), todavía no es claro qué tanto las alteraciones urodinámicas podrían contribuir al problema $a^{30,32,33}$.

En su forma más severa, el síndrome de HinmanAllen (disfunción vesical no neurogénica), hay obstrucción causada por contracciones activas del esfinter vesical externo durante el vaciamiento. Puede conducir una a falla renal y requerir cirugía vesical derivativa ${ }^{34}$.

También existen reportes de hipercalciuria, cistinuria y uricosuria en niños con síndrome de Down $^{23,15,20}$. Incluso se ha reportado una mutación nueva asociada con diabetes insípida nefrogénica congénita ${ }^{35}$. Por último, se conoce que hay un riesgo más bajo de patología neoplásica urológica en las personas con síndrome de Down ${ }^{36}$.

\section{Diagnóstico}

Antenatalmente, se ha planteado un posible beneficio de realizar un análisis cromosómico invasivo, siempre que se detecte uropatía fetal obstructiva $^{9,23}$. Sin embargo, parece ser mejor utilizar el seguimiento ecográfico ${ }^{37}$, junto con la prueba de tamizaje no invasiva ${ }^{35}$.

Luego del nacimiento, sería favorable que todos los pacientes con síndrome de Down confirmado clínica y/o genéticamente, tuviesen una ecografía renal durante la primera semana de vida, dada la posibilidad de hipoplasia/agenesia renal o uropatía obstructiva, en especial cuando no se realizó una ecografía prenatal adecuada ${ }^{38,39}$. Este procedimiento también sería recomendable siempre que se encuentren problemas funcionales ${ }^{30}$. Incluso, por esto mismo, se propone realizarla anualmente ${ }^{14,33}$. Una uretrocistografía miccional debería ser el examen complementario a la ecografía (dado que hay más alteraciones estructurales que funcionales), siguiendo las indicaciones generales de la prueba ${ }^{39}$.

Si hay clínica de incontinencia urinaria o de infecciones urinarias a repetición, o se detecta un RVU o caída de la tasa de filtración glomerular estimada, debería considerarse la existencia de una disfunción vesical funcional asociada y podría ser pertinente una evaluación urológica (uroflujometría o urodinamia $)^{33,40}$. Se ha planteado tener en los requisitos un umbral bajo para beneficiar a más niños con estas pruebas ${ }^{14}$.

La realización de un uroanálisis, y medir las fracciones excretadas de sodio y potasio, la reabsorción tubular de fosfatos, la excreción urinaria de calcio, magnesio, ácido úrico, y el aclaramiento de creatinina y proteinuria, estaría sujeto a los hallazgos de la ecografía o a la aparición de síntomas clínicos de enfermedad renal según las guías específicas de cada patología ${ }^{15}$.

Es conocido que las personas con síndrome de Down padecen más frecuentemente de hiperuricemia y tienen un mayor riesgo de sufrir gota. Aunque no hay claridad sobre el momento en 
el que debería realizarse la medición, posiblemente sea adecuado establecer niveles de ácido úrico si se diagnostica insuficiencia renal ${ }^{20}$.

Dada la etiología variada de las glomerulonefritis en síndrome de Down, no es fácil predecir su curso. Sin embargo, puede conllevar a falla renal terminal con terapia de reemplazo e, incluso, trasplante renal. Por lo tanto, es necesaria la biopsia renal siempre que se considere que los pacientes muestran estas patologías ${ }^{16}$.

Se sabe que la creatinuria y la densidad urinaria son menores en los niños con síndrome de Down, con respecto a los niños sin la condición. Este hallazgo es más notorio conforme aumenta la edad y podría relacionarse con el mayor estrés oxidativo y el envejecimiento prematuro que se observa en estas personas, especialmente cuando son hipotiroideas. No se ha establecido una utilidad específica sobre estos biomarcadores, pero es un elemento que sugiere la necesidad de hacer un seguimiento clínico periódico de la función renal a los pacientes con síndrome de Down ${ }^{15,41,42,43,44}$. Ante la inexistencia de recomendaciones específicas, pareciera razonable hacer una evaluación anual, como en otras patologías.

\section{Enfoque terapéutico}

El manejo de cada patología se basa en sus indicaciones usuales. En los casos quirúrgicos, habrá condicionantes, según las comorbilidades existentes, fundamentalmente cardiovasculares y respiratorias.

Una medida básica de puericultura con los niños con síndrome de Down es establecer un entrenamiento de continencia. Un método sencillo que puede discutirse con los padres está disponible en línea ${ }^{45}$.

Se debe mencionar que la cateterización vesical limpia para la preservación renal y la continencia puede ser realizada en las personas con síndrome de Down, sin importar su compromiso cognitivo ${ }^{34}$. En los casos con enfermedad renal establecida, ya se ha mencionado el seguimiento clínico-ecográfico anual. Los exámenes adicionales se harían con base en esta evaluación.

En la medida de lo posible, se debe ofrecer terapia de reemplazo renal a los niños que lleguen a requerirla (usualmente serán adolescentes o de más edad). En estos casos, parece ser mejor el uso de hemodiálisis, debido a la discapacidad cognitiva de los pacientes ${ }^{46}$. No obstante, si el entorno familiar es adecuado, es posible el uso de diálisis peritoneal ${ }^{13,20,47,48,49,50}$. En los casos que sea factible y beneficioso, se debe ofrecer el trasplante renal con la misma consideración del entorno familiar ${ }^{51,52}$. La tasa de rechazo es similar a otras condiciones ${ }^{20,53,54}$.

\section{Conflicto de intereses}

El autor hace explícito que hace parte del Grupo de Investigación de Síndrome de Down de Santander, el cual no tiene filiación directa con ninguna otra entidad y tampoco posee otra finalidad fuera de la académica, en pro del beneficio de los niños con síndrome de Down. Se declara que no se recibió ningún tipo de remuneración por el desarrollo del presente manuscrito y que no se recibe ninguna contraprestación material por la vinculación con el grupo de investigación mencionado.

\section{Responsabilidades éticas}

\section{Protección de personas y animales}

Los autores declaran que para esta investigación no se han realizado experimentos en seres humanos ni en animales.

\section{Confidencialidad de los datos}

El autor declara que ha seguido los protocolos de su centro de trabajo sobre la publicación de datos de pacientes.

\section{Derecho a la privacidad y consentimiento informado}

Los autores declaran que en este artículo no aparecen datos de pacientes. 


\section{Referencias}

1. World Health Organization. Genes and Human Disease. [Internet] 2013. Disponible en: http://www.who.int/genomics/public/ geneticdiseases/en/index 1.html

2. Nazer H J, Cifuentes O L. Estudio epidemiológico global del síndrome de Down. Rev Chil Pediatr. 2011;82(2):105-112. doi: https://doi.org/10.4067/S0370-41062011000200004

3. Hernández Ramírez I, Manrique Hernández RD. Prevalencia de síndrome de Down en CEHANI-ESE, San Juan de Pasto Colombia. 1998-2003. NOVA. 2006;4(5):50-56. https://doi.org/10.22490/24629448.347

4. Ramírez M RE, Isaza C, Gutiérrez MI. La incidencia del síndrome de Down en Cali. Colombia Médica. 1996;27(3-4):138-142.

5. Madariaga Domínguez L. Anomalías congénitas del riñón y la vía urinaria (CAKUT). [Internet] 2015. Disponible en: https:// continuum.aeped.es/files/guias/Material_descarga_unidad_1_nefrologia.pdf

6. Zarante I, Zarante AM, Fernández N. Frecuencia de malformaciones congénitas genitales y urológicas en Colombia. Rev Arg de Urol. 2009;74(2):85-90.

7. Kupferman J, Druschel C, Kupchik G. Increased Prevalence of Renal and Urinary Tract Anomalies in Children with Down Syndrome. Pediatrics. 2009;124(4):615-621. https://doi.org/10.22490/24629448.34710.1542/peds.2009-0181

8. Morris JK, Garne E, Wellesley D, Addor MC, Arriola L, Barisic I, et al. Major Congenital Anomalies in Babies Born with Down Syndrome: A EUROCAT Population-Based Registry Study. Am J Med Genet A. 2014;164(12):2979-2986. https://doi.org/10.224 90/24629448.34710.1542/peds.2009-018110.1002/ajmg.a.36780

9. Qureshi F, Jacques SM, Feldman B, Doss BJ, Johnson A, Evans MI, Johnson MP. Fetal Obstructive Uropathy in Trisomy Syndromes. Fetal Diagn Ther. 2000;15(6):342-347. https://doi.org/10.1159/000021034

10. de Carvalho Mrad FC, de Bessa J Jr, de Rezende Duarte AM, Vieira AA, Araujo FC, de Sá Camargo ML, et al. Prevalence of Lower Urinary Tract Symptoms in Individuals with Down Syndrome. J Pediatr Urol. 2014;10(5):844-849. https://doi.org/10.1016/j.jpurol.2014.02.011

11. Kapoor S, Bhayana S, Singh A, Kishore J. Co-morbidities Leading to Mortality or Hospitalization in Children with Down Syndrome and its Effect on the Quality of Life of their Parents. Indian J Pediatr. 2014;81(12):1302-1306. https://doi.org/10.1007/ s12098-014-1389-4

12. Bull MJ, The Committee on Genetics. Health Supervision for Children with Down Syndrome. Pediatrics. 2011;128(2):393-406. https://doi.org/10.1542/peds.2011-1605

13. Urbano R. Health Issues Among Persons with Down Syndrome. San Diego: Elsevier; 2010.

14. Ebert AK, Brookman-Amissah S, Rösch WH. Urologische Manifestationen des Down-syndroms: Bedeutung und Langzeitkomplikationen - Eigenes patientengut mit Übersicht. Urologe. 2008;47(3):337-341. https://doi.org/10.1007/s00120-007-1614-0

15. Málaga S, Pardo R, Málaga I, Orejas G, Fernández-Toral J. Renal Involvement in Down Syndrome. Pediatr Nephrol. 2005;20(5):614617. https://doi.org/10.1007/s00467-005-1825-9

16. Said SM, Cornell LD, Sethi S, Fidler ME, Al Masri O, Marple J, Nasr SH. Acquired Glomerular Lesions in Patients with Down Syndrome. Hum Pathol. 2012;43(1):81-88. dhttps://doi.org/10.1016/j.humpath.2011.04.009

17. Lo A, Brown HG, Fivush BA, Neu AM, Racusen LC. Renal Disease in Down Syndrome: Autopsy Study with Emphasis on Glomerular Lesions. Am J Kidney Dis. 1998;31(2):329-335.

18. Assadi FK. IgG-associated mesangial glomerulonephritis in a patient with Down syndrome. Med Sci Monit. 2004;10(9):54-57.

19. Cherif M, Hedri H, Ounissi M, Gergah T, Goucha R, Barbouch S. Pauci-Immune Crescentic Glomerulonephritis in the Down's Syndrome. Saudi J Kidney Dis Transpl. 2013;24(6):1223-1227. https://doi.org/10.4103/1319-2442.121311.

20. Kute VB, Vanikar AV, Shah PR, Gumber MR, Patel HV, Engineer DP, et al. Down Syndrome with End-Stage Renal Disease. Indian J Clin Biochem. 2013;28(4):429-432. doi: 10.1007/s12291-013-0308-1

21. Kusters MA, Verstegen RH, Gemen EF, de Vries E. Intrinsic Defect of the Immune System in Children with Down Syndrome: A Review. Clin Exp Immunol. 2009;156(2):189-193. https://doi.org/10.1111/j.1365-2249.2009.03890.x

22. Haseyama T, Imai H, Komatsuda A, Hamai K, Ohtani H, Kibira S, Miura AB. Proteinase-3-antineutrophil cytoplasmic antibody

58 Anomalías congénitas del riñón y del tracto urinario en niños con síndrome de Down 
(PR3-ANCA) positive crescentic glomerulonephritis in a patient with Down's syndrome and infectious endocarditis. Nephrol Dial Transplant. 1998;13(8):2142-2146.

23. Nickavar A. Polycystic kidney and Down Syndrome. JRMS. 2006;11(4):278-280.

24. Lazarus J, Theron A, Smit S. Posterior urethral valves and Down syndrome. African J Urol. 2015;21(1):4-5.

25. Narasimhan K, Gupta A. Posterior Urethral Valves with Down's Syndrome Presenting as Scrotal Urinary Sinuses. Indian Pediatr. 1993;41(10):1068-1069.

26. Mondal K, Maheshwari A, Aneja S, Seth A. A Case of Down Syndrome with a Posterior Urethral Valve. Indian J Nephrol. 2012;22(5):403-405. https://doi.org/10.4103/0971-4065.103922

27. Narasimhan KL, Kaur B, Marwaha RK. Posterior Urethral Valves in Patients with Down Syndrome. Indian J Pediatr. 2005;72(9):802.

28. Al Harbi NN. Prune-Belly Anomalies in a Girl with Down Syndrome. Pediatr Nephrol. 2003;18(11):1191-1192.

29. Bittles AH, Bower C, Hussain R, Glasson EJ. The Four Ages of Down Syndrome. Eur J Public Health. 2007;17(2):221-225. https://doi.org/10.1093/eurpub/ckl103

30. Hicks JA, Carson C, Malone PS. Is there an Association between Functional Bladder Outlet Obstruction and Down's Syndrome? J Pediatr Urol. 2007;3(5):369-374. https://doi.org/10.1016/j.jpurol.2007.02.003

31. Chicoine B, Sulo S. Rate of Urinary Retention in Adults with Down Syndrome: A Prospective Study. J Am Board Fam Med. 2015;28(1):115-117. https://doi.org/10.3122/jabfm.2015.01.140065.

32. Powers MK, Brown ET, Hogan RM, Martin AD, Ortenberg J, Roth CC. Trends in Toilet Training and Voiding Habits among Children with Down Syndrome. J Urol. 2015;194(3):783-787. https://doi.org/10.1016/j.juro.2015.03.114

33. Seki N, Shahab N. Dysfunctional Voiding of Non-Neurogenic Neurogenic Bladder : A Urological Disorder Associated with Down Syndrome. En Dey S. Genetics and Etiology of Down Syndrome. [Internet] 2011. Disponible en: http://cdn.intechopen.com/pdfs-wm/18447.pdf

34. Handel L, Barqawi A, Checa G, Furness P, Koyle MA. Males with Down's Syndrome and Nonneurogenic Neurogenic Bladder. J Urol. 2003;169(2):646-649. https://doi.org/ 10.1097/01.ju.0000047125.89679.28.

35. Fujisawa Y, Miyamoto T, Furuhashi K, Sano S, Nakagawa Y, Ohzeki T. A Novel Mutation in the Renal V2 Receptor Gene in a Boy with Trisomy 21. Pediatr Nephrol. 2004;19(6):609-611. https://doi.org/10.1007/s00467-004-1446-8

36. Satgé D, Sasco AJ, Day S, Culine S. A Lower Risk of Dying from Urological Cancer in Down Syndrome: Clue for Cancer Protecting Genes on Chromosome 21. Urol Int. 2009;82(3):296-300. https://doi.org/10.1159/000209361

37. Smith-Bindman R, Hosmer W, Feldstein VA, Deeks JJ, Goldberg JD. Second-Trimester Ultrasound to Detect Fetuses with Down Syndrome: A Meta-Analysis. JAMA. 2001;285(8):1044-1055.

38. Madariaga Domínguez L, Ordóñez Álvarez FÁ. Manejo de las anomalías renales y del tracto urinario detectadas por ecografía prenatal. Uropatías obstructivas. Protoc Diagn Ter Pediatr. 2014;1(1):225-239.

39. Jain M, Singh A, Mantan M, Kapoor S. Evaluation of Structural Anomalies of Kidney and Urinary Tract in Children with Down Syndrome. Indian J Pediatr. 2014;81(7):734-734. https://doi.org/10.1007/s12098-013-1244-Z

40. Kitamura A, Kondoh T, Noguchi M, Hatada T, Tohbu S, Mori K, et al. Assessment of Lower Urinary Tract Function in Children with Down Syndrome. Pediatr Int. 2014;56(6):902-908. https://doi.org/ 10.1111/ped.12367

41. Guzmán R, Campos C, López-Fernández E, Casado A. Biomarkers of Age Effect on Renal Function in Down Syndrome. Biomarkers. 2011;16(8):679-85. doi: 10.3109/1354750X.2011.626527

42. Campos C, Guzmán R, López-Fernández E, Casado Á. Evaluation of Urinary Biomarkers of Oxidative/Nitrosative Stress in Adolescents and Adults with Down Syndrome. Biochim Biophys Acta. 2011;1812(7):760-768. https://doi.org/10.1016/j.bbadis.2011.03.013

43. Campos C, Guzmán R, López-Fernández E, Casado Á. Evaluation of Urinary Biomarkers of Oxidative/Nitrosative Stress in Children with Down Syndrome. Life Sci. 2011;89(17-18):655-661. https://doi.org/10.1016/j.lfs.2011.08.006

44. Jovanovic SV, Clements D, MacLeod K. Biomarkers of Oxidative Stress Are Significantly Elevated in Down Syndrome. Free Radic Biol Med. 1998;25(9):1044-1048.

45. National Down Syndrome Society. Toilet Training Children with Down Syndrome. [Internet] 2014 [accedido 18 Ene 2018]. Dis- 
ponible en: http://www.ndss.org/Resources/Therapies-Development/Toilet-Training-Children-with-Down-Syndrome/

46. Kosmadakis G, Smirloglou D, Gobou A, Draganis T, Michail S. Hemodialysis Treatment on an Adult Patient with Down Syndrome Associated with Ectopic Right Kidney Chronic Obstructive Nephropathy and Secondary Amyloidosis. Saudi J Kidney Dis Transpl. 2013;24(2):322-325.

47. Yavascan O, Kara OD, Anil M, Bal A, Pehlivan O, Aksu N. Chronic peritoneal dialysis treatment in a pediatric patient with Down syndrome. Perit Dial Int. 2008;28(5):558-559.

48. Aksu N, Yavascan O, Anil M, Kara OD, Bal A, Anil AB. Chronic Peritoneal Dialysis in Children with Special Needs or Social Disadvantage or Both: Contraindications Are Not Always Contraindications. Perit Dial Int. 2012;32(4):424-430. https://doi. org/10.3747/pdi.2009.00202

49. Horcicka V, Zadrazil J, Zahálková J, Krejcí K, Strebl P, Al Jabry S. A Down syndrome patient treated with peritoneal dialysis. Dial Transplant [Internet]. 2006;35(3):167-75. Available from: http://doi.wiley.com/10.1002/dat.20000

50. Hausmann MJ, Landau D. A Down Syndrome Patient Treated by Peritoneal Dialysis. Nephron. 2002;92(2):484-486. https://doi. org/10.1159/000063317

51. Bagi N, Tejani A, Sullivan E. Renal transplantation in Down syndrome: a report of the North American Pediatric Renal Transplant Cooperative Study. Pediatr Transpl. 1998;2(3):211-215.

52. Knoll G, Cockfield S, Blydt-Hansen T, Baran D, Kiberd B, Landsberg D, Cole E. Canadian Society of Transplantation Consensus Guidelines on Eligibility for Kidney Transplantation. CMAJ. 2005;173(10):S1-25. https://doi.org/10.1503/cmaj.051291

53. Wightman A, Young B, Bradford M, Dick A, Healey P, McDonald R, Smith J. Prevalence and outcomes of renal transplantation in children with intellectual disability. Pediatr Transplant. 2014;18(7):714-719. https://doi.org/10.1111/petr.12339

54. Galante NZ, Dib GA, Medina-Pestana JO. Severe Intellectual Disability Does Not Preclude Renal Transplantation. Nephrol Dial Transplant. 2010;25(8):2753-2757. https://doi.org/10.1093/ndt/gfq105 